\title{
Stability Analysis for Stochastic Markovian Jump Reaction-Diffusion Neural Networks with Partially Known Transition Probabilities and Mixed Time Delays
}

\author{
Weiyuan Zhang, ${ }^{1,2}$ Junmin $\mathrm{Li}^{1}{ }^{1}$ and Naizheng Shi ${ }^{1}$ \\ ${ }^{1}$ School of Science, Xidian University, Shaanxi, Xi'an 710071, China \\ ${ }^{2}$ Institute of Mathematics and Applied Mathematics, Xianyang Normal University, Shaanxi, \\ Xianyang 712000, China \\ Correspondence should be addressed to Weiyuan Zhang, ahzwy@163.com
}

Received 11 January 2012; Accepted 28 February 2012

Academic Editor: Josef Diblík

Copyright (C) 2012 Weiyuan Zhang et al. This is an open access article distributed under the Creative Commons Attribution License, which permits unrestricted use, distribution, and reproduction in any medium, provided the original work is properly cited.

\begin{abstract}
The stability problem is proposed for a new class of stochastic Markovian jump reaction-diffusion neural networks with partial information on transition probability and mixed time delays. The new stability conditions are established in terms of linear matrix inequalities (LMIs). To reduce the conservatism of the stability conditions, an improved Lyapunov-Krasovskii functional and freeconnection weighting matrices are introduced. The obtained results are dependent on delays and the measure of the space AND, therefore, have less conservativeness than delay-independent and space-independent ones. An example is given to show the effectiveness of the obtained results.
\end{abstract}

\section{Introduction}

During the last decades, neural networks (NNs) with time delays have received considerable attention, because time delays existed in many fields, for instance, finite switching speeds of amplifiers and transmission of signals in a network, which affect the system performance [1-4]. Therefore, the stability analysis of NNs with time delays has attracted more and more attention of the researchers. Various sufficient conditions, either delay-dependent or delayindependent, have been derived to ensure the stability for NNs [1-3]. On the other hand, Markovian jump systems (MJSs) involve both time-evolving and event-driven mechanisms, which can be employed to model the abrupt phenomena such as random failures and repairs of the components, changes in the interconnections of subsystems, and sudden environment changes. The issues of stability, stabilization, control, and filtering for the systems have 
been well investigated [5-8]. In such systems, the transition probabilities of the jumping process are crucial, almost all the issues on MJSs have been investigated assuming the complete knowledge of these transition probabilities. However, in most cases the transition probabilities of MJSs are not exactly known. Whether in theory or in practice, it is necessary to further consider more general MJSs with partial information on transition probabilities. Recently, considerable attention has been devoted to the research on MJSs with partial information on transition probability [9-11].

As it is well known, the diffusion phenomena could not be ignored in NNs and electric circuits when electrons are moving in a nonuniform electromagnetic field. Therefore, it is essential to consider the state variables varying with the time and space variables. The NNs with diffusion terms can commonly be expressed by partial differential equations [12-28]. In the real world, a real system is usually affected by unknown disturbances, which may be regarded as stochastic processes [20, 24, 29, 30]. Consequently, it is of significant importance to study stochastic effects for the NNs. In recent years, the dynamic behaviors of stochastic NNs, especially the stability of stochastic NNs, have become a hot study topic. Recently, several kinds of NNs with delays and stochastic effects have been investigated [20, 24, 29]. For example, the authors in [20] have obtained some criteria to guarantee the almost sure exponential stability, and mean square exponential stability of an equilibrium solution for reaction-diffusion neural networks (RDNNs) with continuously distributed delays and stochastic influence. However, in these papers, due to the complicated behavior for stochastic Markovian jump RDNNs with partial information on transition probability and mixed time delays, very few results on such systems have appeared.

Based on the above discussions, we are interested in the asymptotic stability of the equilibrium point in the mean square sense for a new class of stochastic Markovian jump RDNNs with partial information on transition probability and mixed time delays. To the best of our knowledge, till now, this is the first attempt to introduce and investigate stochastic Markovian jump RDNNs with partial information on transition probability and mixed time delays. In this paper, the contribution of our note is two-fold. Firstly, the proposed RDNNs systems will be more general and cover the cases of systems with completely unknown or known transition probabilities. Secondly, based on the Lyapunov stability theory combined with linear matrix inequality (LMI) technique some novel stability conditions in terms of LMIs are derived by introducing some free-connection weighting matrices which can be selected properly to lead much less conservative results. Some asymptotic stability criteria are dependent on delays and the measure of the space, therefore, they have less conservativeness than delay-independent and space-independent ones. Furthermore, the results depend on the reaction-diffusion terms. The criteria of our paper are new and they complement previously known results. Hence, it is shown that the newly obtained results are less conservative and more applicable than the existing corresponding ones. An example is given to illustrate the effectiveness of the proposed method.

Notation. In this paper, the superscript " $T$ " stands for matrix transposition; $\mathbb{R}^{n}$ denotes the $n$-dimensional Euclidean space. Vector $X \in \mathbb{R}^{n}$, its norm is defined as $|X|=\sqrt{X^{T} X}$. For symmetric matrices $A$ and $B$, the notation $A>B(A \geq B)$ means that the $A-B$ is positive definite (semipositive-definite). The symmetric terms in a symmetric matrix are denoted by $*$. Mathematical expectation will be denoted by $E[\cdot] \cdot \operatorname{tr}(\cdot)$ denotes the trace of the corresponding matrix. 
Discrete Dynamics in Nature and Society

For $u(t, x)=\left(u_{1}(t, x), u_{2}(t, x), \ldots, u_{n}(t, x)\right)^{T} \in \mathbb{R}^{n}$, we define

$$
\|u(t, x)\|_{2}=\sum_{i=1}^{n}\left\|u_{i}(t, x)\right\|_{2}=\sum_{i=1}^{n}\left[\int_{\Omega}\left|u_{i}(t, x)\right|^{2} d x\right]^{1 / 2} .
$$

Let $P \mathbb{C}\left[(-\widetilde{\tau}, 0] \times \Omega ; \mathbb{R}^{n}\right]$ denote the family of right continuous function $\varphi$ from $(-\widetilde{\tau}, 0] \times \Omega$ to $\mathbb{R}^{n}$ with the uniform norm $\|\varphi(s, x)\|_{\tilde{\tau}}=\sup _{-\tilde{\tau} \leq s \leq 0} \sum_{i=1}^{n}\left\|\varphi_{i}(s, x)\right\|_{2}$. Denote by $L_{F_{t}}^{2}[(-\tilde{\tau}, 0] \times$ $\left.\Omega ; \mathbb{R}^{n}\right]$ the family of all $F_{t}$ measurable, $P \mathbb{C}\left[(-\tilde{\tau}, 0] \times \Omega ; \mathbb{R}^{n}\right]$-valued stochastic variables $\xi=$ $\{\xi(\theta, x): \theta \in(-\tilde{\tau}, 0]\}$ such that $\int_{\Omega} \int_{-\widetilde{\tau}}^{0} E|\xi(s, x)|^{2} d s d x<\infty$.

\section{Problem Formulation and Preliminaries}

In this paper, we consider the following stochastic Markovian jumping RDNNs with partial information on transition probability and mixed time delays:

$$
\begin{aligned}
& d u(t, x)=\sum_{l=1}^{m} \frac{\partial}{\partial x_{l}}\left(D \frac{\partial u(t, x)}{\partial x_{l}}\right) d t \\
& +[-A(r(t)) u(t, x)+B(r(t)) f(u(t, x)) \\
& \left.+C(r(t)) g(u(t-d(t), x))+E(r(t)) \int_{t-\tau(t)}^{t} h(u(s, x)) d s+J\right] d t \\
& +\sigma\left(t, x, u(t, x), u(t-d(t), x), \int_{t-\tau(t)}^{t} h(u(s, x)) d s, r(t)\right) d w(t), \\
& t \geq t_{0} \geq 0, \quad x \in \Omega, \quad u(t, x)=0, \quad(t, x) \in[0,+\infty) \times \partial \Omega, \\
& u\left(t_{0}+s, x\right)=\varphi(s, x), \quad(s, x) \in(-\tilde{\tau}, 0] \times \bar{\Omega},
\end{aligned}
$$

where $\{r(t), t \geq 0\}$ is a right-continuous Markovian chain on the probability space which takes values in the finite space $S=\{1,2, \ldots, N\}$ with generator $\Gamma=\left(\gamma_{i j}\right)_{N \times N}$ given by

$$
P\{r(t+\delta)=j \mid r(t)=i\}= \begin{cases}\gamma_{i j} \delta+o(\delta), & \text { if } i \neq j, \\ 1+\gamma_{i j} \delta+o(\delta), & \text { if } i=j,\end{cases}
$$

with $\delta>0$ and $\lim _{\delta \rightarrow 0} O(\delta) / \delta=0, \gamma_{i j} \geq 0$ is the transition rate from $i$ to $j$ if $i \neq j$ and $\gamma_{i i}=$ $-\sum_{i \neq j} \gamma_{i j}$. Assume that $x=\left(x_{1}, x_{2}, \ldots, x_{m}\right)^{T} \in \Omega, \Omega$ is a compact set with smooth boundary $\partial \Omega$ and mes $\Omega>0$ in space $\mathbb{R}^{m}, \bar{\Omega}=\partial \Omega \cup \Omega ; u(t, x)=\left(u_{1}(t, x), \ldots, u_{n}(t, x)\right)^{T} \in \mathbb{R}^{n}$ denotes the state vector associated with the $n$ neurons at time $t$ and in space $x$; the diagonal matrix $A(r(t))=$ $\operatorname{diag}\left(a_{1}(r(t)), . ., a_{n}(r(t))\right)$ has positive entries $a_{i}(r(t))>0, B(r(t))=\left(b_{i j}(r(t))\right)_{n \times n^{\prime}} C(r(t))=$ $\left(c_{i j}(r(t))\right)_{n \times n}$ and $E(r(t))=\left(e_{i j}(r(t))\right)_{n \times n}$ are the interconnection matrices representing the weight coefficients of the neurons; $f(u(t, x))=\left(f_{1}\left(u_{1}(t, x)\right), \ldots, f_{n}\left(u_{n}(t, x)\right)\right)^{T}, g(u(t, x))=$ $\left(g_{1}\left(u_{1}(t, x)\right), \ldots, g_{n}\left(u_{n}(t, x)\right)\right)^{T}$ and $h(u(s, x))=\left(h_{1}\left(u_{1}(s, x)\right), \ldots, h_{n}\left(u_{n}(s, x)\right)\right)^{T}$ are the neuron 
activation functions, and $J=\left(J_{1}, J_{2}, \ldots, J_{n}\right)^{T}$ denotes a constant external input vector. $d(t)$ and $\tau(t)$ denote the discrete time-varying delay and the distributed time-varying delay, respectively. Time-delays are assumed to satisfy $0 \leq d(t) \leq d_{0}, 0 \leq \tau(t) \leq \tau_{0}$, where $d_{0}$ and $\tau_{0}$ are positive constants, $\max \left\{\tau_{0}, d_{0}\right\}=\tilde{\tau}, \dot{\tau}(t) \leq \mu<1, \dot{d}(t) \leq d<1$, where $d$ and $\mu$ are constants; $D=\operatorname{diag}\left(D_{1}, D_{2}, \ldots, D_{n}\right) D_{i}=D_{i}(t, x, u) \geq 0, i=1,2, \ldots n$ stand for transmission diffusion operator along the $i$ th neurons. The noise perturbation $\sigma(\cdot)=\sigma(t, x, u(t, x), u(t-$ $\left.d(t), x), \int_{t-\tau(t)}^{t} h(u(s, x)) d s, r(t)\right)$ is the noise intensity matrix; $w(t)=\left(w_{1}(t), \ldots, w_{n}(t)\right)^{T}$ is an $n$-dimensional standard Brownian motion defined on a complete probability space $\left(\bar{\Omega}, F,\left\{F_{t}\right\}_{t \geq 0}, P\right)$ with a filtration $\left\{F_{t}\right\}_{t \geq 0}$ generated by $\{w(s): 0 \leq s \leq t\}$, where we associate $\bar{\Omega}$ with the canonical space generated by all $\left\{w_{i}(t)\right\}$ and denote by $F$ the associated $\sigma$-algebra generated by $\{w(t)\}$ with the probability measure $P$. Moreover, we assume that the Brownian motion $\{w(t): t \geq 0\}$ is independent from the Markov chain $\{r(t): t \geq 0\} . \bar{n}$ is the outer normal vector of $\partial \Omega, \varphi_{i}(s, x)$ is given bounded and continuous on $(-\widetilde{\tau}, 0] \times \Omega, i, j=1,2, \ldots, n$.

Since the transition probability depends on the transition rates for the continuous-time MJSs, the transition rates of the jumping process are considered to be partly available, namely, some elements in matrix $\Theta$ are unknown in this paper. For example, the transition rate matrix $\Theta$ for system (2.1) with $N$ operation modes may be expressed as

$$
\Theta=\left[\begin{array}{ccccc}
\gamma_{11} & ? & \gamma_{13} & \ldots & \gamma_{1 N} \\
? & ? & \gamma_{23} & \ldots & \gamma_{2 N} \\
\vdots & \vdots & \vdots & \ddots & \vdots \\
? & \gamma_{N 2} & ? & \ldots & \gamma_{N N}
\end{array}\right]
$$

where "?" represents the unknown transition rate. For notation clarity, for all $i \in S$, the set $\Xi^{i}$ denotes $\Xi^{i}=\Xi_{k}^{i} \cup \Xi_{u k}^{i}$ with $\Xi_{k}^{i}=\left\{j: \gamma_{i j}\right.$ is known for $\left.j \in S\right\}, \Xi_{u k}^{i}=\left\{j: \gamma_{i j}\right.$ is unknown for $j \in S\}$.

Furthermore, when $\Xi_{k}^{i} \neq \phi$, it is further described as

$$
\Xi_{k}^{i}=\left\{k_{1}^{i}, k_{2}^{i} \ldots, k_{n}^{i}\right\}
$$

where $1 \leq n \leq N, n \in N^{*}$ and $k_{j}^{i} \in N^{*}, 1 \leq k_{j}^{i} \leq N, j=1,2, \ldots, n$ represent the $j$ th known element of the set $\Xi_{k}^{i}$ in the $i$ th row of the transition rate matrix $\Theta$.

Remark 2.1. It is noted that the jumping process is commonly assumed to be completely available $\left(\Xi_{u k}^{i}=\phi, \Xi_{k}^{i}=\Xi^{i}\right)$ or completely unavailable $\left(\Xi_{u k}^{i}=\Xi^{i}, \Xi_{k}^{i}=\phi\right)$. However, in most cases the transition probabilities of MJSs are not exactly known. Whether in theory or in practice, it is necessary to further consider more general jump systems with partial information on transition probabilities. Recently, a considerable amount of attention has been paid to studying the stability and stabilization of general MJSs governed by ordinary differential equations with partly unknown transition probabilities [9-11]. It is well known that the stability analysis of partial differential equations with partial information on transition probabilities is more complicated, very few results on such systems have appeared. In this paper, the new stability criteria for a new class of novel stochastic Markovian jump RDNNs with partial information on transition probability and mixed time delays are investigated. 
For the sake of simplicity, we write $r(t)=i \in S$, and

$$
\begin{aligned}
d u(t, x)= & \sum_{l=1}^{n} \frac{\partial}{\partial x_{l}}\left(D \frac{\partial u(t, x)}{\partial x_{l}}\right) d t \\
& +\left[-A_{i} u(t, x)+B_{i} f(u(t, x))+C_{i} g(u(t-d(t), x))+E_{i} \int_{t-\tau(t)}^{t} h(u(s, x)) d s+J\right] d t \\
& +\sigma_{i}\left(t, x, u(t, x), u(t-d(t), x), \int_{t-\tau(t)}^{t} h(u(s, x)) d s\right) d w(t), \quad t \geq t_{0} \geq 0, x \in \Omega,
\end{aligned}
$$

Throughout this paper, the following assumptions are made.

(A1) There exist positive diagonal matrices $L^{f}=\operatorname{diag}\left(L_{1}^{f}, \ldots, L_{n}^{f}\right), L^{g}=\operatorname{diag}\left(L_{1}^{g}, \ldots, L_{n}^{g}\right), L^{h}=$ $\operatorname{diag}\left(L_{1}^{h}, \ldots, L_{n}^{h}\right)$, such that

$$
0 \leq \frac{f_{j}\left(\xi_{1}\right)-f_{j}\left(\xi_{2}\right)}{\xi_{1}-\xi_{2}} \leq L_{j}^{f}, \quad 0 \leq \frac{g_{j}\left(\xi_{1}\right)-g_{j}\left(\xi_{2}\right)}{\xi_{1}-\xi_{2}} \leq L_{j}^{g}, \quad 0 \leq \frac{h_{j}\left(\xi_{1}\right)-h_{j}\left(\xi_{2}\right)}{\xi_{1}-\xi_{2}} \leq L_{j}^{h},
$$

for all $\xi_{1}, \xi_{2} \in \mathbb{R}, \xi_{1} \neq \xi_{2}, j=1,2, \ldots, n$.

(A2) There exist diagonal matrices $\sum_{i 1}, \sum_{i 2}$ and $\sum_{i 3}(i \in S)$ such that

$$
\operatorname{tr}\left[\left(\sigma_{i}\left(t, x, \zeta_{1}, \zeta_{2}, \zeta_{3}\right)\right)^{T}\left(\sigma_{i}\left(t, x, \zeta_{1}, \zeta_{2}, \zeta_{3}\right)\right)\right] \leq\left|\sum_{i 1} \zeta_{1}\right|^{2}+\left|\sum_{i 2} \zeta_{2}\right|^{2}+\left|\sum_{i 3} \zeta_{3}\right|^{2}
$$

for all $\zeta_{1}, \zeta_{2}, \zeta_{3} \in \mathbb{R}^{n}$

(A3) There exist constants $D_{i}^{*}$ such that $D_{i} \geq D_{i}^{*} \geq 0, i=1,2, \ldots, n$.

(A4) $\sigma_{i}\left(t, x, u^{*}, u^{*}, \int_{t-\tau(t)}^{t} h\left(u^{*}(s, x)\right) d s\right)=0$, where $u^{*}$ is the equilibrium point of system (2.1).

Remark 2.2. By assumption (A1)-(A4), it is not difficult to prove that there exists a unique equilibrium point $u^{*}$ for system (2.1) based on Mawhin's continuation theorem [31]. 
The main aim of this paper is to investigate the asymptotic stability in the mean square sense of system (2.1). Let $u^{*}=\left(u_{1}^{*}, u_{2}^{*}, \ldots, u_{n}^{*}\right)^{T}$ be the equilibrium point of system (2.1). Now, we set $z(t) \triangleq u(t, x)-u^{*}$, which yields the following system:

$$
\begin{gathered}
d z(t)=\sum_{l=1}^{n} \frac{\partial}{\partial x_{l}}\left(D \frac{\partial z(t)}{\partial x_{l}}\right) d t \\
+\left[-A_{i} z(t)+B_{i} \bar{f}(z(t))+C_{i} \bar{g}(z(t-d(t)))+E_{i} \int_{t-\tau(t)}^{t} \bar{h}(z(s)) d s\right] d t \\
+\sigma_{i}\left(t, x, z(t), z(t-d(t)), \int_{t-\tau(t)}^{t} \bar{h}(z(s)) d s\right) d w(t), \quad t \geq t_{0}, \quad x \in \Omega, \\
z(t)=0, \quad t \geq t_{0}, \quad x \in \partial \Omega, \quad z\left(t_{0}+s\right)=\varphi(s)-u^{*}, \\
(s, x) \in(-\tilde{\tau}, 0] \times \bar{\Omega},
\end{gathered}
$$

where $\bar{f}(z(t))=f\left(z(t)+u^{*}\right)-f\left(u^{*}\right), \bar{g}(z(t))=g\left(z(t)+u^{*}\right)-g\left(u^{*}\right), \bar{h}(z(s))=h(z(s)+$ $\left.u^{*}\right)-h\left(u^{*}\right), \bar{f}(z(t))=\left(\bar{f}_{1}\left(z_{1}(t)\right), \ldots, \bar{f}_{n}\left(z_{n}(t)\right)\right)^{T}, \bar{g}(z(t))=\left(\bar{g}_{1}\left(z_{1}(t)\right), \ldots, \bar{g}_{n}\left(z_{n}(t)\right)\right)^{T}, \bar{h}(z(s))=$ $\left(\bar{h}_{1}\left(z_{1}(s)\right), \ldots, \bar{h}_{n}\left(z_{n}(s)\right)\right)^{T}$.

Clearly, $\bar{f}_{j}\left(z_{j}(t)\right), \bar{g}_{j}\left(z_{j}(t)\right)$, and $\bar{h}_{j}\left(z_{j}(s)\right)$ satisfy (A1). Thus, the stability problem of system (2.1) is equivalent to the stability problem of system (2.8).

Definition 2.3. The equilibrium point $u^{*}=\left(u_{1}^{*}, u_{2}^{*}, \ldots, u_{n}^{*}\right)^{T}$ of system (2.1) is said to be asymptotic stability in the mean square sense if there exists a constant $\delta>0$ and for any $\varepsilon>0$ satisfying that $E\left\|u(t, x)-u^{*}\right\|_{2}^{2}<\varepsilon$ and $\lim _{t \rightarrow \infty} E\left\|u(t, x)-u^{*}\right\|_{2}^{2}=0$ when $t \geq t_{0} \geq 0$ and $\left\|\varphi(s, x)-u^{*}\right\|_{\tau}<\delta$, where $\left\|\varphi-u^{*}\right\|_{\tau}=\sup _{-\tilde{\tau} \leq s \leq 0}\left\|\varphi(s, x)-u^{*}\right\|_{2}$.

Lemma 2.4 (see [2] (Jensen inequality)). Consider the following. (i) For any constant matrix $\Xi \in$ $\mathbb{R}^{n \times n}, \Xi>0$, scalar function $d(t): 0<d(t)<d$, and vector function $\omega:[0, d] \rightarrow \mathbb{R}^{n}$ such that the integrations concerned are well defined, then

$$
\left(\int_{0}^{d(t)} \omega(s) d s\right)^{T} \Xi\left(\int_{0}^{d(t)} \omega(s) d s\right) \leq d(t) \int_{0}^{d(t)} \omega(s)^{T} \Xi \omega(s) d s
$$

(ii) For any constant matrix $\Xi \in R^{n \times n}, \Xi>0, \Omega \subset \mathbb{R}^{n}$, mes $\Omega>0$, if $\omega: \Omega \rightarrow \mathbb{R}^{n}$ is vector function such that the integration is well defined, then

$$
\left(\int_{\Omega} \omega(s) d s\right)^{T} \Xi\left(\int_{\Omega} \omega(s) d s\right) \leq|\Omega| \int_{\Omega} \omega(s)^{T} \Xi \omega(s) d s
$$


Lemma 2.5 (see [24]). Let $\Omega$ be a bounded open domain in $\mathbb{R}^{m}$ with smooth boundary $\partial \Omega$. If $h=$ $h(x)$ define on $\Omega$ is a smooth function with $\left.h\right|_{\partial \Omega}=0$, then the following inequality holds:

$$
\int_{\Omega} h^{2}(x) d x \leq \alpha \int_{\Omega}\left(\frac{\partial h(x)}{\partial x}\right)^{2} d x
$$

where $|\Omega|$ denotes the volume of $\Omega, \alpha=\left(|\Omega| / \omega_{m}\right)^{1 / m}>0, \omega_{m}$ denotes the surface area of unit ball in $\mathbb{R}^{m}$.

\section{Main Results}

Theorem 3.1. Under assumptions (A1)-(A4), if there exist positive definite diagonal matrices $P_{i}$, $\Lambda_{i}=\Lambda_{i}^{T}$, positive definite symmetry matrices $Q_{1}, Q_{2}, G, W, K_{1}, K_{2}$, positive definite diagonal matrices $M_{1}, M_{2}$ with appropriate dimensions, and scalar $\rho_{i}>0$, such that the following Linear matrix inequalities (LMIs) hold:

$$
\begin{gathered}
\Xi_{1}=\left[\begin{array}{cccccccc}
\alpha_{11} & 0 & 0 & \alpha_{14} & \alpha_{15} & \alpha_{16} & 0 & 0 \\
* & \alpha_{22} & 0 & 0 & 0 & 0 & 0 & 0 \\
* & * & \alpha_{33} & 0 & 0 & 0 & 0 & 0 \\
* & * & * & \alpha_{44} & 0 & 0 & 0 & 0 \\
* & * & * & * & \alpha_{55} & 0 & 0 & 0 \\
* & * & * & * & * & \alpha_{66} & 0 & 0 \\
* & * & * & * & * & * & \alpha_{77} & 0 \\
* & * & * & * & * & * & * & \alpha_{88}
\end{array}\right]<0, \\
P_{j}-\Lambda_{i} \leq 0, \quad j \in \rho_{i} I, \\
P_{i}-\Lambda_{i} \geq 0, \quad j \in \Xi_{u k^{\prime}}^{i} \quad j \neq i, \\
\end{gathered}
$$

where

$$
\begin{gathered}
\alpha_{11}=-P_{i} A_{i}-A_{i} P_{i}-P_{i} R-R P_{i}+Q_{1}+Q_{2}+L^{g} G L^{g}+\sum_{j \in \Xi_{k}^{i}} \gamma_{i j}\left(P_{j}-\Lambda_{i}\right) \\
\quad+\rho_{i} \sum_{i 1}^{T} \sum_{i 1}+\tau_{0} L^{h} W L^{h}+\tau_{0}|\Omega| L^{h} K_{1} L^{h}+d_{0}|\Omega| L^{g} K_{2} L^{g}, \\
\alpha_{14}=P_{i} B_{i}+L^{f} M_{1}^{T}, \quad \alpha_{15}=P_{i} C_{i}, \quad \alpha_{16}=P_{i} E_{i},
\end{gathered}
$$




$$
\begin{aligned}
& \alpha_{22}=-(1-\mu) Q_{1}, \quad \alpha_{33}=L^{g} M_{2} L^{g}+\rho_{i} \sum_{i 2}^{T} \sum_{i 2}-(1-d) Q_{2}, \\
& \alpha_{44}=-2 M_{1}, \quad \alpha_{55}=-(1-d) G-M_{2}, \quad \alpha_{66}=\rho_{i} \sum_{i 3}^{T} \sum_{i 3}-\tau_{0} W, \\
& \alpha_{77}=-\tau_{0}^{-1} K_{1}, \quad \alpha_{88}=-d_{0}^{-1} K_{2},
\end{aligned}
$$

$R=\operatorname{diag}\left\{-D_{1}^{*} / \alpha, \ldots,-D_{n}^{*} / \alpha\right\}$, then equilibrium point $u^{*}$ of system (2.1) with a partly known transition rate matrix (2.3) is an asymptotic stability in the mean square sense.

Proof. Consider the Lyapunov-Krasovskii functional:

$$
\begin{aligned}
V(t, z(t), i)= & \int_{\Omega} z(t)^{T} P_{i} z(t) d x+\int_{\Omega} \int_{t-\tau(t)}^{t} z(s)^{T} Q_{1} z(s) d s d x \\
& +\int_{\Omega} \int_{t-d(t)}^{t} z(s)^{T} Q_{2} z(s) d s d x+\int_{\Omega} \int_{t-d(t)}^{t} \bar{g}(z(s))^{T} G \bar{g}(z(s)) d s d x \\
& +\int_{\Omega} \int_{-\tau_{0}}^{0} \int_{t+\theta}^{t} \bar{h}(z(s))^{T} W \bar{h}(z(s)) d s d \theta d x \\
& +\int_{-d_{0}}^{0} \int_{t+\theta}^{t}\left(\int_{\Omega} \bar{g}(z(s)) d x\right)^{T} K_{2}\left(\int_{\Omega} \bar{g}(z(s)) d x\right) d s d \theta \\
& +\int_{-\tau_{0}}^{0} \int_{t+\theta}^{t}\left(\int_{\Omega} \bar{h}(z(s)) d x\right)^{T} K_{1}\left(\int_{\Omega} \bar{h}(z(s)) d x\right) d s d \theta
\end{aligned}
$$

Define an operator $L$ associated with $(2.8)$ acting on $V(t, z(t), i)$ by

$$
\begin{aligned}
L V(t, z(t), i)= & V_{t}(t, z(t), i)+V_{z}(t, z(t), i) \\
& \times\left[\sum_{l=1}^{n} \frac{\partial}{\partial x_{l}}\left(D \frac{\partial z(t)}{\partial x_{l}}\right) d t-A_{i} z(t)+B_{i} \bar{f}(z(t))+C_{i} \bar{g}(z(t-d(t)))\right. \\
& \left.\quad+E_{i} \int_{t-\tau(t)}^{t} \bar{h}(z(s)) d s\right]+\frac{1}{2} \operatorname{trace}\left[\sigma_{i}^{T}(\cdot) V_{z z}(t, z, i) \sigma_{i}(\cdot)\right]+\sum_{j=1}^{N} \gamma_{i j} V(t, z(t), j),
\end{aligned}
$$

where $V_{t}(t, z(t), i)=\partial V(t, z(t), i) / \partial t, V_{z}(t, z(t), i)=(\partial V(t, z(t), i)) / \partial z_{1}, \ldots,(\partial V(t, z(t), i)) / \partial z_{n}$, $V_{z z}(t, z(t), i)=\left(\partial^{2} V(t, z(t), i) / \partial z_{i} \partial z_{j}\right)_{n \times n}$. 
The weak infinitesimal generator $L V(t, z, i)$ along with (2.8), leads to

$$
\begin{aligned}
L V(t, z, i)= & \int_{\Omega}\left\{2 z(t)^{T} P_{i}\left(\frac{\partial z(t)}{\partial t}\right)+\sum_{j=1}^{N} \gamma_{i j} z(t)^{T} P_{j} z(t)+\operatorname{trace}\left[\sigma_{i}^{T}(\cdot) P_{i} \sigma_{i}(\cdot)\right]\right\} d x \\
& +\int_{\Omega}\left[z(t)^{T} Q_{1} z(t)-(1-\dot{\tau}(t)) z(t-\tau(t))^{T} Q_{1} z(t-\tau(t))\right] d x \\
& +\int_{\Omega}\left[z(t)^{T} Q_{2} z(t)-(1-\dot{d}(t)) z(t-d(t))^{T} Q_{2} z(t-d(t))\right] d x \\
& +\int_{\Omega}\left[\bar{g}(z(t))^{T} G \bar{g}(z(t))-(1-\dot{d}(t)) \bar{g}(z(t-d(t)))^{T} G \bar{g}(z(t-d(t)))\right] d x \\
& +\int_{\Omega}\left[\tau_{0} \bar{h}(z(t))^{T} W \bar{h}(z(t))-\int_{t-\tau(t)}^{t} \bar{h}(z(s))^{T} W \bar{h}(z(s)) d s\right] d x \\
& +\int_{-\tau_{0}}^{0}\left(\int_{\Omega} \bar{h}(z(t)) d x\right)^{T} K_{1}\left(\int_{\Omega} \bar{h}(z(t)) d x\right) d \theta \\
& -\int_{-\tau_{0}}^{0}\left(\int_{\Omega} \bar{h}(z(t+\theta)) d x\right)^{T} K_{1}\left(\int_{\Omega} \bar{h}(z(t+\theta)) d x\right) d \theta \\
& +\int_{-d_{0}}^{0}\left(\int_{\Omega} \bar{g}(z(t)) d x\right)^{T} K_{2}\left(\int_{\Omega} \bar{g}(z(t)) d x\right) d \theta \\
& -\int_{-d_{0}}^{0}\left(\int_{\Omega} \bar{g}(z(t+\theta)) d x\right)^{T} K_{2}\left(\int_{\Omega} \bar{g}(z(t+\theta)) d x\right) d \theta .
\end{aligned}
$$

Considering the situation that the information of transition probabilities is not accessible completely, the following equalities satisfy for arbitrary matrices $\Lambda_{i}=\Lambda_{i}^{T}$, due to $\sum_{j=1}^{N} \gamma_{i j}=0$

$$
-z(t)^{T} \sum_{j=1}^{N} \gamma_{i j} \Lambda_{i} z(t)=0
$$

From (A1), we have

$$
\begin{gathered}
\int_{\Omega} \bar{f}(z(t))^{T} M_{1} L^{f} z(t)-\bar{f}(z(t))^{T} M_{1} \bar{f}(z(t)) d x \geq 0, \\
\int_{\Omega} z(t-d(t))^{T} L^{g} M_{2} L^{g} z(t-d(t))-\bar{g}(z(t-d(t)))^{T} M_{2} \bar{g}(z(t-d(t))) d x \geq 0,
\end{gathered}
$$

where $M_{1}$ and $M_{2}$ are positive definite diagonal matrices. 
According to Lemma 2.4, we derive

$$
\begin{aligned}
& \int_{-\tau_{0}}^{0}\left(\int_{\Omega} \bar{h}(z(t)) d x\right)^{T} K_{1}\left(\int_{\Omega} \bar{h}(z(t)) d x\right) d \theta \\
& -\int_{-\tau_{0}}^{0}\left(\int_{\Omega} \bar{h}(z(t+\theta)) d x\right)^{T} K_{1}\left(\int_{\Omega} \bar{h}(z(t+\theta)) d x\right) d \theta \\
& \leq \tau_{0}|\Omega| \int_{\Omega} \bar{h}(z(t))^{T} K_{1} \bar{h}(z(t)) d x \\
& -\tau_{0}^{-1}\left(\int_{t-\tau(t)}^{t} \int_{\Omega} \bar{h}(z(s)) d x d s\right)^{T} K_{1}\left(\int_{t-\tau(t)}^{t} \int_{\Omega} \bar{h}(z(s)) d x d s\right), \\
& \int_{-d_{0}}^{0}\left(\int_{\Omega} \bar{g}(z(t)) d x\right)^{T} K_{2}\left(\int_{\Omega} \bar{g}(z(t)) d x\right) d \theta \\
& -\int_{-d_{0}}^{0}\left(\int_{\Omega} \bar{g}(z(t+\theta)) d x\right)^{T} K_{2}\left(\int_{\Omega} \bar{g}(z(t+\theta)) d x\right) d \theta \\
& \leq d_{0}|\Omega| \int_{\Omega} \bar{g}(z(t))^{T} K_{2} \bar{g}(z(t)) d x \\
& -d_{0}^{-1}\left(\int_{t-d(t)}^{t} \int_{\Omega} \bar{g}(z(s)) d x d s\right)^{T} K_{2}\left(\int_{t-d(t)}^{t} \int_{\Omega} \bar{g}(z(s)) d x d s\right), \\
& -\int_{\Omega} \int_{t-\tau(t)}^{t} \bar{h}(z(s))^{T} W \bar{h}(z(s)) d s d x \\
& \leq-\tau_{0}^{-1} \int_{\Omega}\left[\int_{t-\tau(t)}^{t} \bar{h}(z(s)) d s\right]^{T} W\left[\int_{t-\tau(t)}^{t} \bar{h}(z(s)) d s\right] d x .
\end{aligned}
$$

According to the boundary condition, $D_{i} \geq 0$ and Lemma 2.5, applying Green's formula, we get

$$
\begin{aligned}
\int_{\Omega} \sum_{l=1}^{m} z_{i}(t) \frac{\partial}{\partial x_{l}}\left(D_{i} \frac{\partial z_{i}(t)}{\partial x_{l}}\right) d x & =\sum_{l=1}^{m} \int_{\Omega} \frac{\partial}{\partial x_{l}}\left(D_{i} \frac{\partial z_{i}(t)}{\partial x_{l}} z_{i}(t)\right) d x-\sum_{l=1}^{m} \int_{\Omega} D_{i}\left(\frac{\partial z_{i}(t)}{\partial x_{l}}\right)^{2} d x \\
& =\int_{\partial \Omega} D_{i} z_{i}(t) \frac{\partial z_{i}(t)}{\partial v} d s-\sum_{l=1}^{m} \int_{\Omega} D_{i}\left(\frac{\partial z_{i}(t)}{\partial x_{l}}\right)^{2} d x \\
& \leq-\frac{D_{i}^{*}}{\alpha} \int_{\Omega} z_{i}(t)^{2} d x
\end{aligned}
$$

where $\alpha=\left(|\Omega| / \omega_{m}\right)^{1 / m}>0$. 
From (3.6)-(3.11), we have

$$
\begin{aligned}
& L V(t, z(t), i) \leq \int_{\Omega} z(t)^{T}\left(-P_{i} A_{i}-A_{i} P_{i}-P_{i} R-R P_{i}+Q_{1}+Q_{2}+L^{g} G L^{g}+\sum_{j \in \Xi_{k}^{i}} \gamma_{i j}\left(P_{j}-\Lambda_{i}\right)\right. \\
& \left.+\rho_{i} \sum_{i 1}^{T} \sum_{i 1}+\tau_{0} L^{h} W L^{h}+\tau_{0}|\Omega| L^{h} K_{1} L^{h}+d_{0}|\Omega| L^{g} K_{2} L^{g}\right) z(t) d x \\
& +\int_{\Omega} z(t)^{T}\left(P_{i} B_{i}+L^{f} M_{1}^{T}\right) \bar{f}(z(t)) d x \\
& +\int_{\Omega} z(t)^{T} \sum_{j \in \Xi_{u k}^{i}} \gamma_{i j}\left(P_{j}-\Lambda_{i}\right) z(t) d x+\int_{\Omega} \bar{f}(z(t))^{T}\left(B_{i}^{T} P_{i}+M_{1} L^{f}\right) z(t) d x \\
& +\int_{\Omega} z(t)^{T}\left(P_{i} C_{i}\right) \bar{g}(z(t-d(t))) d x+\int_{\Omega} \bar{g}(z(t-d(t)))^{T}\left(C_{i}^{T} P_{i}\right) z(t) d x \\
& +\int_{\Omega} z(t)^{T} P_{i} E_{i} \int_{t-\tau(t)}^{t} \bar{h}(z(s)) d s d x+\int_{\Omega}\left(\int_{t-\tau(t)}^{t} \bar{h}(z(s)) d s\right)^{T} E_{i}^{T} P_{i} z(t) d x \\
& +\int_{\Omega} z(t-\tau(t))^{T}\left[-(1-\mu) Q_{1}\right] z(t-\tau(t)) d x \\
& -\int_{\Omega} \bar{g}(z(t-d(t)))^{T}\left[(1-d) G+M_{2}\right] \bar{g}(z(t-d(t))) d x \\
& -2 \int_{\Omega} \bar{f}(z(t))^{T} M_{1} \bar{f}(z(t)) d x+\int_{\Omega} z(t-d(t))^{T} \\
& \times\left(L^{g} M_{2}^{T} L^{g}+\rho_{i} \sum_{i 2}^{T} \sum_{i 2}-(1-d) Q_{2}\right) z(t-d(t)) d x \\
& +\int_{\Omega}\left(\int_{t-\tau(t)}^{t} \bar{h}(z(s)) d s\right)^{T}\left(\rho_{i} \sum_{i 3}^{T} \sum_{i 3}-\tau_{0}^{-1} W\right)\left(\int_{t-\tau(t)}^{t} \bar{h}(z(s)) d s\right) d x \\
& -\tau_{0}^{-1}\left(\int_{t-\tau(t)}^{t} \int_{\Omega} \bar{h}(z(s)) d x d s\right)^{T} K_{1}\left(\int_{t-\tau(t)}^{t} \int_{\Omega} \bar{h}(z(s)) d x d s\right) \\
& -d_{0}^{-1}\left(\int_{t-d(t)}^{t} \int_{\Omega} \bar{g}(z(s)) d x d s\right)^{T} K_{2}\left(\left(\int_{t-d(t)}^{t} \int_{\Omega} \bar{g}(z(s)) d x d s\right)\right) \\
& =\int_{\Omega} \varsigma_{1}^{T} \Xi_{1} \varsigma_{1} d x
\end{aligned}
$$


where

$$
\begin{aligned}
\varsigma_{1}= & \left(z(t)^{T}, z(t-\tau(t))^{T} z(t-d(t))^{T}, \bar{f}(z(t))^{T} \bar{g}(z(t-d(t)))^{T}\right. \\
& \left.\left(\int_{t-\tau(t)}^{t} \bar{h}(z(s)) d s\right)^{T}\left(\int_{t-\tau(t)}^{t} \int_{\Omega} \bar{h}(z(s)) \mathrm{d} x d s\right)^{T}\left(\int_{t-d(t)}^{t} \int_{\Omega} \int \bar{g}(z(s)) \mathrm{d} x d s\right)^{T}\right)^{T} .
\end{aligned}
$$

From the conditions of Theorem 3.1, noting that $\gamma_{i i}=-\sum_{j=1, j \neq i}^{N} \gamma_{i j}$ and $\gamma_{i j} \geq 0$ for all $j \neq i$, that is $\gamma_{i i}<0$ for all $i \in S$. Hence, when $i \in \Xi_{k^{\prime}}^{i}$, from (3.1) and $\varsigma_{1} \neq 0$, we can derive

$$
L V(t, z(t), i)<0
$$

On the other hand, if $i \in \Xi_{u k}^{i}$, according to (3.1) and $\varsigma_{1} \neq 0$, we have (3.14) holds.

Therefore, if $\left\|u\left(t_{0}, x\right)-u^{*}\right\|_{2}<\delta$ and $k \rightarrow \infty, \lim _{t \rightarrow \infty} E\left\|u(t, x)-u^{*}\right\|_{2}=0$, which implies that system (2.1) is asymptotic stability in the mean square sense. This completes the proof.

Remark 3.2. In proof of above Theorem 3.1, the new Lyapunov functional to construct is more general. It is easy to see that our results expanded the model in [23]. The obtained results in [23] are independent on the measure of the space and diffusion effects. The results of this paper are dependent on the measure of the space and diffusion effects. Besides delays and stochastic effects, it is necessary to further consider more general MJSs with partial information on transition probabilities. Searching for stability analysis issue for stochastic Markovian jump RDNNs with partial information on transition probability and mixed time delays is obviously more preferable and challenging. However, no such results have been available up to now. Moreover, in this paper, the idea of free-connection weighting matrix is introduced, this method may lead to derive an improved feasible region for delay-dependent and space-dependent stability criteria. Therefore, it is shown that the newly obtained results are less conservative and more applicable than the existing corresponding ones.

Remark 3.3. In $[8,26]$, free weighting matrix was introduced via Newton Leibniz formula and system transformation, respectively. Theorems 3.2 and 4.1 in [26] and Corollary 3.2 in [8] have no restriction on the derivative of time-varying delays. The same way can be directly applied into our paper to cancel the restricted on the derivative of the time-varying delays. In order to simplify and avoid repeating the technique in [26], we do not do so. While new free-connection weighting matrices are introduced in (3.6) by using the relationship of the transition rates among various subsystems, that is, $\sum_{j=1}^{N} \gamma_{i j}=0$, for all $i \in S$, in this paper, which may develop less-conservative results. This is quite different from free weighting matrices in $[8,26]$, we introduced free-connection weighting matrices lead to the obtained criteria are dependent on delays, the measure of the space and the reaction-diffusion terms, therefore, have less conservativeness than delay-independent, space-independent, and diffusion terms independent ones [15, 22, 23, 25], respectively. 
When the smooth operator $D_{i}=0, i=1,2, \ldots, n$, model (2.2) is reduced to the following model:

$$
\begin{gathered}
d u(t)=\left[-A_{i} u(t)+B_{i} f(u(t))+C_{i} g(u(t-d(t)))+E_{i} \int_{t-\tau(t)}^{t} h(u(s)) d s+J\right] d t \\
+\sigma_{i}\left(t, u(t), u(t-d(t)), \int_{t-\tau(t)}^{t} h(u(s)) d s\right) d w(t), \quad t \geq t_{0} \geq 0, \\
u\left(t_{0}+s\right)=\varphi(s), \quad s \in(-\tilde{\tau}, 0] .
\end{gathered}
$$

Remark 3.4. The system (3.15) is a special case of system (2.1). In [32], the authors consider the following model:

$$
\dot{u}=-A u+B f(u(t))+C g(u(t-d(t)))+E \int_{t-\tau(t)}^{t} h(u(s)) d s+J .
$$

However, To the best of our knowledge, the LMI technique to guarantee the asymptotic stability of stochastic Markovian jump NNs with partial information on transition probability and discrete and distributed time-varying delays is not studied which is very important in both theories and applications and is also a very challenging problem.

From (3.15), we have the following corollary.

Corollary 3.5. Suppose that, $(A 1)-(A 2)$ and $(A 4)$ hold. If there exist positive definite diagonal matrices $P_{i}, \Lambda_{i}=\Lambda_{i}^{T}$, positive definite symmetry matrices $Q_{1}, Q_{2}, G, W, K_{1}, K_{2}$, positive definite diagonal matrices $M_{1}, M_{2}$ with appropriate dimensions, and scalar $\rho_{i}>0$, such that the following LMIs hold:

$$
\begin{gathered}
\Xi_{2}=\left[\begin{array}{ccccccc}
\bar{\alpha}_{11} & 0 & 0 & \alpha_{14} & \alpha_{15} & \alpha_{16} & 0 \\
* & \alpha_{22} & 0 & 0 & 0 & 0 & 0 \\
* & * & \alpha_{33} & 0 & 0 & 0 & 0 \\
* & * & * & \alpha_{44} & 0 & 0 & 0 \\
* & * & * & * & \alpha_{55} & 0 & 0 \\
* & * & * & * & * & \bar{\alpha}_{66} & 0 \\
* & * & * & * & * & * & \bar{\alpha}_{77}
\end{array}\right]<0, \\
P_{i} \leq \rho_{i} I, \\
P_{j}-\Lambda_{i} \leq 0, \quad j \in \Xi_{u k^{\prime}}^{i} \quad j \neq i \\
P_{i}-\Lambda_{i} \geq 0, \quad j \in \Xi_{u k^{\prime}}^{i} \quad j=i
\end{gathered}
$$

where $\bar{\alpha}_{11}=-P_{i} A_{i}-A_{i} P_{i}-P_{i} R-R P_{i}+Q_{1}+Q_{2}+L^{g} G L^{g}+\sum_{j \in U_{k}^{i}} \gamma_{i j}\left(P_{j}-\Lambda_{i}\right)+\rho_{i} \sum_{i 1}^{T} \sum_{i 1}+$ $\tau_{0} L^{h} K_{1} L^{h}+d_{0} L^{g} K_{2} L^{g}, \bar{\alpha}_{66}=\rho_{i} \sum_{i 3}^{T} \sum_{i 3}-\tau_{0}^{-1} K_{1}, \bar{\alpha}_{77}=-d_{0}^{-1} K_{2}$, the other notations are the same as those in Theorem 3.1, then system (3.15) with a partly unknown transition rate matrix (2.3) is asymptotic stability in the mean square sense. 
Proof. By constructing the following Lyapunov functional:

$$
\begin{aligned}
V(t, z(t), i)= & z(t)^{T} P_{i} z(t)+\int_{t-\tau(t)}^{t} z(s)^{T} Q_{1} z(s) d s \\
& +\int_{t-d(t)}^{t} z(s)^{T} Q_{2} z(s) d s+\int_{t-d(t)}^{t} \bar{g}(z(s))^{T} G \bar{g}(z(s)) d s \\
& +\int_{-d_{0}}^{0} \int_{t+\theta}^{t} \bar{g}(z(s))^{T} K_{2} \bar{g}(z(s)) d s d \theta \\
& +\int_{-\tau_{0}}^{0} \int_{t+\theta}^{t}(\bar{h}(z(s)))^{T} K_{1}(\bar{h}(z(s))) d s d \theta
\end{aligned}
$$

Similarly, calculate the derivative along the system (3.15). By a minor modification of the proof of Theorem 3.1, we can easily obtain that system (3.15) is asymptotic stability in the mean square sense.

\section{An Illustrative Example}

In this section, we provide the effectiveness of the proposed stability criteria through solving a numerical example. Here, we consider the system (2.1) with three modes described as

$$
\begin{gathered}
A_{1}=\left[\begin{array}{cc}
6 & 0.1 \\
0.6 & 13
\end{array}\right], \quad A_{2}=\left[\begin{array}{ll}
15 & 0.6 \\
0.7 & 10
\end{array}\right], \quad A_{3}=\left[\begin{array}{ll}
10 & 0.5 \\
0.6 & 11
\end{array}\right], \\
B_{1}=\left[\begin{array}{ll}
0.1 & 0.2 \\
0.2 & 0.1
\end{array}\right], \quad B_{2}=\left[\begin{array}{ll}
0.5 & 0.3 \\
0.5 & 0.1
\end{array}\right], \quad B_{3}=\left[\begin{array}{ll}
0.3 & 0.4 \\
0.2 & 0.1
\end{array}\right], \\
C_{1}=C_{2}=C_{3}=\left[\begin{array}{cc}
0.5 & 0 \\
0 & 0.5
\end{array}\right], \\
E_{1}=E_{2}=E_{3}=\left[\begin{array}{cc}
0.1 & 0 \\
0 & 0.1
\end{array}\right], \\
\Sigma_{11}=\Sigma_{12}=\Sigma_{13}=1.2 I, \quad \Sigma_{21}=\Sigma_{22}=\Sigma_{23}=0.8 I, \quad \Sigma_{31}=\Sigma_{32}=\Sigma_{33}=1.1 I, \\
\tau_{0}=d_{0}=0.6, \quad \mu=d=0.2, \quad|\Omega|=\omega_{m}=\pi, \\
L^{f}=L^{g}=L^{h}=I, \quad D_{1}=D_{2}=D_{3}=D_{1}^{*}=D_{2}^{*}=D_{3}^{*}=1, \quad R=-I .
\end{gathered}
$$

The transition probability matrices of form (2.5) are given by

$$
\Theta=\left[\begin{array}{ccc}
-0.3 & ? & ? \\
? & 0.1 & ? \\
? & ? & 0.15
\end{array}\right]
$$


In (3.1), by applying the MATLAB LMI Control Toolbox, we obtain the feasible solution as follows:

$$
\begin{gathered}
P_{1}=\left[\begin{array}{cc}
1.9466 & 0 \\
0 & 1.9466
\end{array}\right], \quad P_{2}=\left[\begin{array}{cc}
1.9072 & 0 \\
0 & 1.9072
\end{array}\right], \quad P_{3}=\left[\begin{array}{cc}
1.7064 & 0 \\
0 & 1.7064
\end{array}\right], \\
Q_{1}=\left[\begin{array}{ll}
1.3124 & 0.1123 \\
0.1123 & 3.3850
\end{array}\right], \quad Q_{2}=\left[\begin{array}{ll}
5.1903 & 0.1019 \\
0.1019 & 7.1036
\end{array}\right], \quad G=\left[\begin{array}{ll}
0.8189 & 0.0984 \\
0.0984 & 2.6072
\end{array}\right], \\
\Lambda_{1}=\left[\begin{array}{cc}
3.6542 & 0.3005 \\
0.3005 & 22.2842
\end{array}\right] \quad \Lambda_{2}=\left[\begin{array}{cc}
0.9530 & -0.0010 \\
-0.0010 & 0.9875
\end{array}\right], \quad \Lambda_{3}=\left[\begin{array}{ll}
0.8493 & 0.0014 \\
0.0014 & 0.8382
\end{array}\right], \\
W=\left[\begin{array}{ll}
5.2683 & 0.1424 \\
0.1424 & 7.9714
\end{array}\right], \quad K_{1}=\left[\begin{array}{ll}
0.6909 & 0.0599 \\
0.0599 & 1.8031
\end{array}\right], \quad K_{2}=\left[\begin{array}{ll}
0.7028 & 0.0597 \\
0.0597 & 1.8035
\end{array}\right], \\
A_{1}=\left[\begin{array}{cc}
3.9211 & 0 \\
0 & 3.9211
\end{array}\right], \quad A_{2}=\left[\begin{array}{cc}
1.2151 & 0 \\
0 & 1.2151
\end{array}\right] .
\end{gathered}
$$

Therefore, it follows from Theorem 3.1 that system (2.1) is asymptotic stability in the mean square sense.

\section{Conclusions}

In this paper, we have dealt with an interesting and important problem of asymptotic stability in the mean square sense for a new class of stochastic Markovian jump RDNNs with unknown transition rates and mixed time delays based on LMI and free-connection weighting matrix techniques. By applying the Lyapunov stability analysis approach, the novel stability criteria for that system are proposed. The obtained criteria are dependent on delays, the measure of the space, and the reaction-diffusion terms, therefore, they have less conservativeness than delay-independent, space-independent, and diffusion terms independent ones, respectively. So, it is shown that the newly obtained results are less conservative and more applicable than the existing corresponding ones. A numerical example has been given to show the effectiveness of the obtained results.

\section{Acknowledgments}

This work is partially supported by the National Natural Science Foundation of China under Grant no. 60974139 and the Fundamental Research Funds for the Central Universities no. 72103676.

\section{References}

[1] J. H. Park and H. J. Cho, "A delay-dependent asymptotic stability criterion of cellular neural networks with time-varying discrete and distributed delays," Chaos, Solitons E Fractals, vol. 33, no. 2, pp. 436442, 2007. 
[2] K. Gu, V. Kharitonov, and J. Chen, Stability of Time-Delay Systems, Birkhäuser, Boston, Mass, USA, 2003.

[3] J. Cao and J. Wang, "Global asymptotic and robust stability of recurrent neural networks with time delays," IEEE Transactions on Circuits and Systems. I, vol. 52, no. 2, pp. 417-426, 2005.

[4] J. Diblík, D. Ya. Khusainov, I. V. Grytsay, and Z. Šmarda, "Stability of nonlinear autonomous quadratic discrete systems in the critical case," Discrete Dynamics in Nature and Society, Article ID 539087, 23 pages, 2010.

[5] H. Zhang and Y. Wang, "Stability analysis of Markovian jumping stochastic Cohen-Grossberg neural networks with mixed time delays," IEEE Transactions on Neural Networks, vol. 19, no. 2, pp. 366-370, 2008.

[6] S. Xu, J. Lam, and X. Mao, “Delay-dependent $H_{\infty}$ control and filtering for uncertain Markovian jump systems with time-varying delays," IEEE Transactions on Circuits and Systems. I, vol. 54, no. 9, pp. 2070-2077, 2007.

[7] M. Rưžičková and I. Dzhalladova, "The optimization of solutions of the dynamic systems with random structure," Abstract and Applied Analysis, vol. 2011, Article ID 486714, 18 pages, 2011.

[8] P. Balasubramaniam and V. Vembarasan, "Robust stability of uncertain fuzzy BAM neural networks of neutral-type with Markovian jumping parameters and impulses," Computers $\mathcal{E}$ Mathematics with Applications, vol. 62, no. 4, pp. 1838-1861, 2011.

[9] L. Zhang and E.-K. Boukas, "Stability and stabilization of Markovian jump linear systems with partly unknown transition probabilities," Automatica, vol. 45, no. 2, pp. 463-468, 2009.

[10] L. Zhang, E.-K. Boukas, and J. Lam, "Analysis and synthesis of Markov jump linear systems with time-varying delays and partially known transition probabilities," IEEE Transactions on Automatic Control, vol. 53, no. 10, pp. 2458-2464, 2008.

[11] Y. Zhang, Y. He, M. Wu, and J. Zhang, "Stabilization for Markovian jump systems with partial information on transition probability based on free-connection weighting matrices," Automatica, vol. 47, no. 1, pp. 79-84, 2011.

[12] J. Pan, X. Liu, and S. Zhong, "Stability criteria for impulsive reaction-diffusion Cohen-Grossberg neural networks with time-varying delays," Mathematical and Computer Modelling, vol. 51, no. 9-10, pp. 1037-1050, 2010.

[13] Z. Wang, H. Zhang, and P. Li, "An LMI approach to stability analysis of reactiondiffusion cohengrossberg neural networks concerning dirichlet boundary conditions and distributed delays," IEEE Transactions on Systems, Man, and Cybernetics, Part B, vol. 40, no. 6, pp. 1596-1606, 2010.

[14] Q. Luo, F. Deng, J. Bao, and B. Zhao, "Sliding mode control of a class of Itô type distributed parameter systems with delay," Acta Mathematica Scientia, Series B, vol. 27, no. 1, pp. 67-76, 2007.

[15] J. Qiu and J. Cao, "Delay-dependent exponential stability for a class of neural networks with time delays and reaction-diffusion terms," Journal of the Franklin Institute, vol. 346, no. 4, pp. 301-314, 2009.

[16] Q. Song, J. Cao, and Z. Zhao, "Periodic solutions and its exponential stability of reaction-diffusion recurrent neural networks with continuously distributed delays," Nonlinear Analysis: Real World Applications, vol. 7, no. 1, pp. 65-80, 2006.

[17] L. Wang and D. Xu, "Global exponential stability of Hopfield reaction-diffusion neural networks with time-varying delays," Science in China, Series F, vol. 46, no. 6, pp. 466-474, 2003.

[18] J. G. Lu, "Robust global exponential stability for interval reaction-diffusion Hopfield neural networks with distributed delays," IEEE Transactions on Circuits and Systems II, vol. 54, no. 12, pp. 1115-1119, 2007.

[19] Q. Zhu, X. Li, and X. Yang, "Exponential stability for stochastic reaction-diffusion BAM neural networks with time-varying and distributed delays," Applied Mathematics and Computation, vol. 217, no. 13, pp. 6078-6091, 2011.

[20] X. Xu, J. Zhang, and W. Zhang, "Mean square exponential stability of stochastic neural networks with reaction-diffusion terms and delays," Applied Mathematics Letters, vol. 24, no. 1, pp. 5-11, 2011.

[21] X. Zhang, S. Wu, and K. Li, “Delay-dependent exponential stability for impulsive Cohen-Grossberg neural networks with time-varying delays and reaction-diffusion terms," Communications in Nonlinear Science and Numerical Simulation, vol. 16, no. 3, pp. 1524-1532, 2011.

[22] L. Wang, R. Zhang, and Y. Wang, "Global exponential stability of reaction-diffusion cellular neural networks with S-type distributed time delays," Nonlinear Analysis: Real World Applications, vol. 10, no. 2, pp. 1101-1113, 2009.

[23] L. Wang, Z. Zhang, and Y. Wang, "Stochastic exponential stability of the delayed reaction-diffusion recurrent neural networks with Markovian jumping parameters," Physics Letters. A, vol. 372, no. 18, pp. 3201-3209, 2008. 
[24] L. Teng and P. Yan, "Dynamical behaviors of reaction-diffusion fuzzy neural networks with mixed delays and general boundary conditions," Communications in Nonlinear Science and Numerical Simulation, vol. 16, no. 2, pp. 993-1001, 2011.

[25] P. Balasubramaniam and C. Vidhya, "Global asymptotic stability of stochastic BAM neural networks with distributed delays and reaction-diffusion terms," Journal of Computational and Applied Mathematics, vol. 234, no. 12, pp. 3458-3466, 2010.

[26] P. Balasubramaniam and M. Syed Ali, "Robust stability of uncertain fuzzy cellular neural networks with time-varying delays and reaction diffusion terms," Neurocomputing, vol. 74, no. 1-3, pp. 439-446, 2010.

[27] W. Zhang and J. Li, "Global exponential stability of reaction-diffusion neural networks with discrete and distributed time-varying delays," Chinese Physics B, vol. 20, no. 3, Article ID 030701, 2011.

[28] W. Zhang and J. Li, "Global exponential synchronization of delayed BAM neural networks with reaction-diffusion terms and the Neumann boundary conditions," Boundary Value Problems, vol. 2012, article 2, 2012.

[29] P. Balasubramaniam, and C. Vidhya, "Exponential stability of stochastic reaction-diffusion uncertain fuzzy neural networks with mixed delays and Markovian jumping parameters," Expert Systems with Applications, vol. 39, pp. 3109-3115, 2012.

[30] I. A. Dzhalladova, J. Baštinec, J. Diblík, and D. Y. Khusainov, “Estimates of exponential stability for solutions of stochastic control systems with delay," Abstract and Applied Analysis, vol. 2011, Article ID 920412, 14 pages, 2011.

[31] X. Lou and B. Cui, "Stochastic stability analysis for delayed neural networks of neutral type with Markovian jump parameters," Chaos, Solitons \& Fractals, vol. 39, no. 5, pp. 2188-2197, 2009.

[32] T. Li, Q. Luo, C. Sun, and B. Zhang, "Exponential stability of recurrent neural networks with timevarying discrete and distributed delays," Nonlinear Analysis: Real World Applications, vol. 10, no. 4, pp. 2581-2589, 2009. 


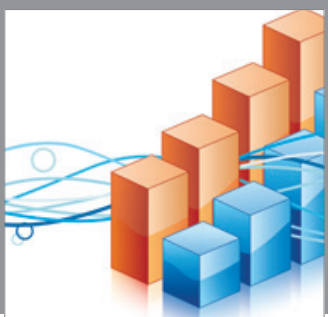

Advances in

Operations Research

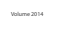

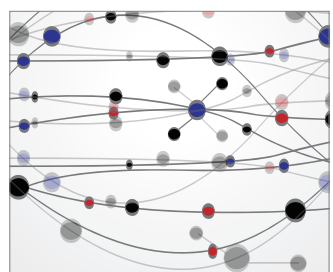

\section{The Scientific} World Journal
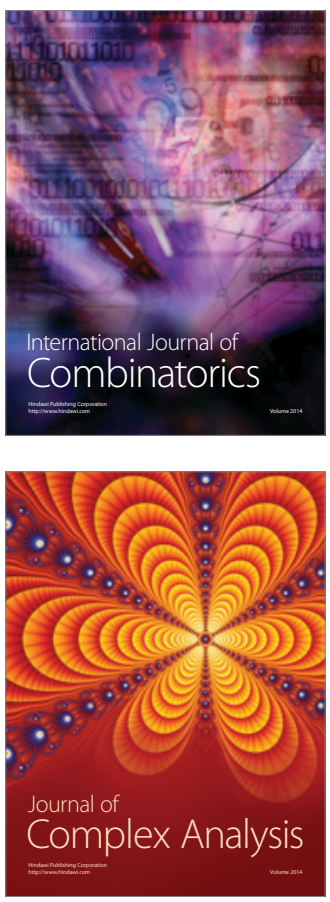

International Journal of

Mathematics and

Mathematical

Sciences
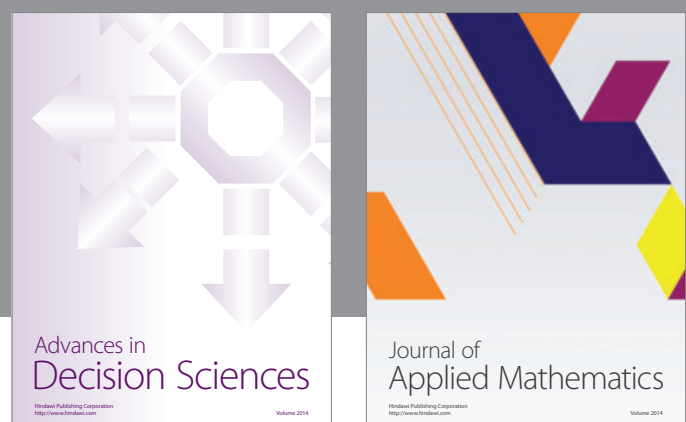

Journal of

Applied Mathematics
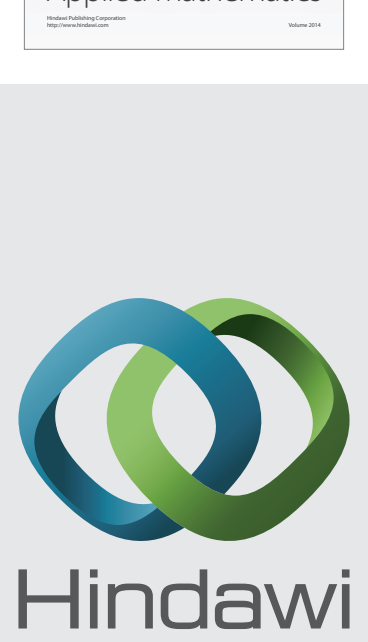

Submit your manuscripts at http://www.hindawi.com
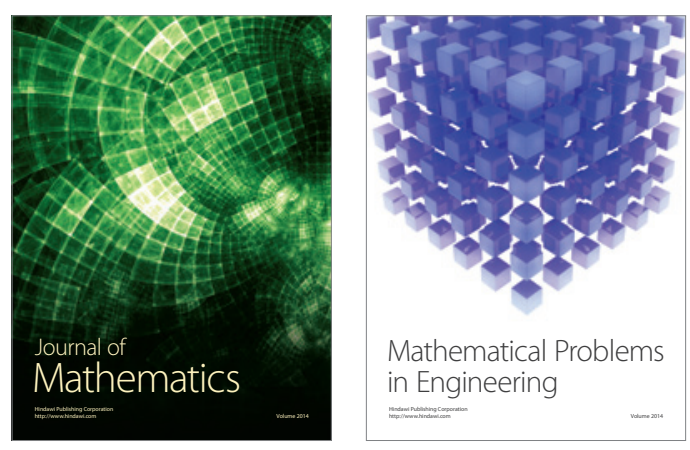

Mathematical Problems in Engineering
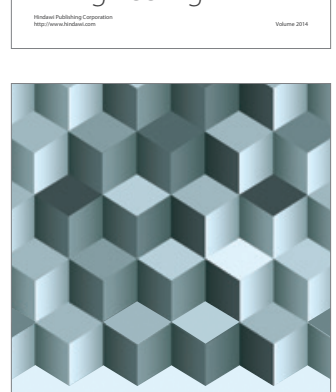

Journal of

Function Spaces
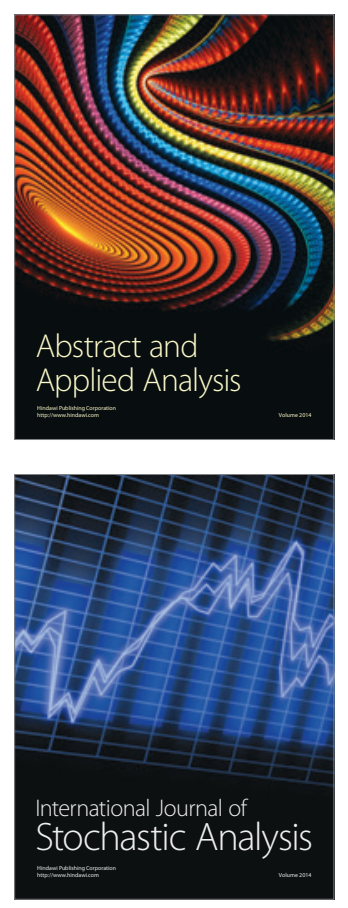

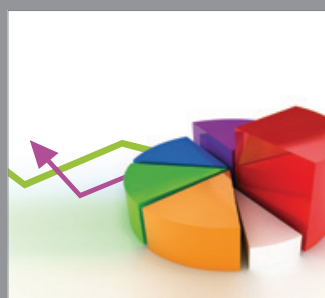

ournal of

Probability and Statistics

Promensencen
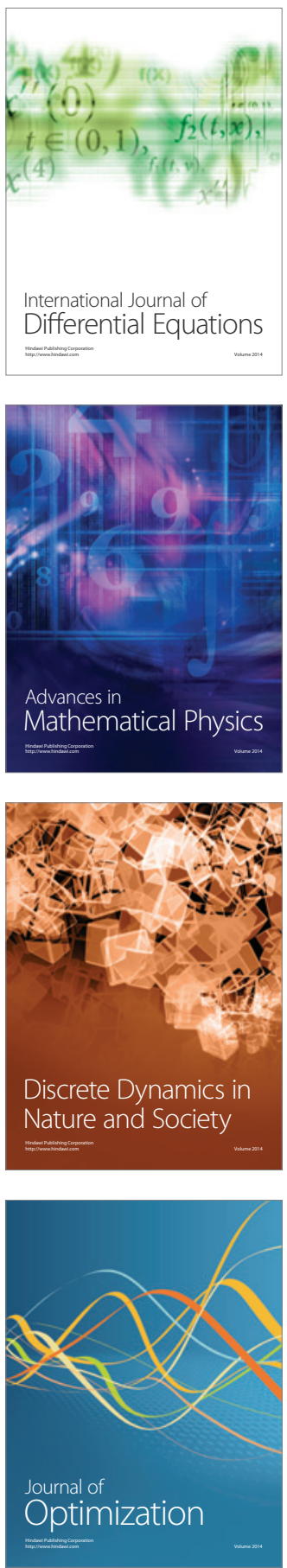